\title{
Approximating Solutions of Non Linear First Order Abstract Measure Differential Equations by Using Dhage Iteration Method
}

\section{Dnyanoba Maroti Suryawanshi, Sidheshwar Sangram Bellale, Pratiksha Prakash Lenekar}

Dayanand Science College Latur, Swami Ramanand Teerth Marathwada University, Nanded, India

Email address:

dmsuryawanshi24@gmail.com (D. M. Suryawanshi), sidhesh.bellale@gmail.com (S. S. Bellale)

\section{To cite this article:}

Dnyanoba Maroti Suryawanshi, Sidheshwar Sangram Bellale, Pratiksha Prakash Lenekar. Approximating Solutions of Non Linear First Order Abstract Measure Differential Equations by Using Dhage Iteration Method. Applied and Computational Mathematics.

Vol. 9, No. 3, 2020, pp. 64-69. doi: 10.11648/j.acm.20200903.13

Received: April 15, 2020; Accepted: April 30, 2020; Published: June 4, 2020

\begin{abstract}
In this paper we have proved the approximating solutions of the nonlinear first order abstract measure differential equation by using Dhage's iteration method. The main result is based on the iteration method included in the hybrid fixed point theorem in a partially ordered normed linear space. Also we have solved an example for the applicability of given results in the paper. Sharma [2] initiated the study of nonlinear abstract differential equations and some basic results concerning the existence of solutions for such equations. Later, such equations were studied by various authors for different aspects of the solutions under continuous and discontinuous nonlinearities. The study of fixed point theorem for contraction mappings in partial ordered metric space is initiated by different authors. The study of hybrid fixed point theorem in partially ordered metric space is initiated by Dhage with applications to nonlinear differential and integral equations. The iteration method is also embodied in hybrid fixed point theorem in partially ordered spaces by Dhage [12]. The Dhage iteration method is a powerful tool for proving the existence and approximating results for nonlinear measure differential equations. The approximation of the solutions are obtained under weaker mixed partial continuity and partial Lipschitz conditions. In this paper we adopted this iteration method technique for abstract measure differential equations.
\end{abstract}

Keywords: Abstract Measure Differential Equation, Dhage Iteration Method, Existence Theorem, Extremal Solutions, Approximation of Solution, Hybrid Fixed Point Theorem

\section{Introduction}

The abstract measure differential equations involve the derivative of the unknown set-function with respect to the

$\sigma$-finite complete measure. Some of the abstract measure differential equations have been studied in a series of papers by Joshi [3], Shendge and Joshi [4], Dhage [14, 15], Dhage et al. [6] and Dhage and Bellale [9, 10] and Suryawanshi and bellale [17] for different aspects of the solutions. The fixed point theorems so far used in the above papers of Dhage [15], Joshi [6], Bellale [13] study the abstract measure integro differential equation and existence theorem. This is a required condition and recently, the authors in Dhage [16], Suryawanshi and Bellale [18] have proved the existence and uniqueness results for abstract measure differential equations. Here our approach is different from that of Sharma [2] and Joshi [3].
The results of this paper complement and generalize the results of the above-mentioned papers on abstract measure differential equations under weaker conditions.

The perturbed ordinary differential equations have been treated in Krasnoselskii [1] and it is mentioned that the inverse of such equations gets the sum of two operators in appropriate function spaces. The Krasnoselskii [1] fixed point theorem is useful for proving the existence results for such perturbed differential equations under mixed geometrical and topological conditions on the nonlinearities involved in them.

\section{Preliminaries}

A mapping if there exists a continuous and non-decreasing function $\phi: R^{+} \rightarrow R^{+}$such that 
$\|T x-T y\| \leq \phi(\|x-y\|)$ for all $x, y \in X$, where $\phi(0)=0$. In particular if $\phi(r)=\alpha r, \alpha>0, T$ is called a Lipschitz function with a Lipschitz constant $\alpha$. Further if $\alpha<1$, then $T$ is called a contraction on $X$ with the contraction constant $\alpha$.

Let $\mathrm{X}$ be a Banach space and let $T: X \rightarrow X, \mathrm{~T}$ is called compact if $\overline{T(X)}$ is a compact subset of X.T is called totally bounded if for any bounded subset $S$ of $X, T(S)$ is a bounded subset of X.T is called completely continuous if $\mathrm{T}$ is continuous and bounded on X. Every compact operator is totally bounded, but the converse may not be true, however, two notions are equivalent on bounded subsets of $\mathrm{X}$. The details of different types of nonlinear contraction, compact and completely continuous operators appear in Granas and Dugundji [8].

\section{Statement of the Problem}

Let $X$ be a real Banach algebra with a convenient norm $\|$.$\| . Let x, y \in X$. Then the line segment $\overline{x y}$ in $X$ is defined by

$$
\overline{x y}=\{z \in X \mid z=x+r(y-x), 0 \leq r \leq 1\}
$$

Let $x_{0} \in X$ be a fixed point and $z \in X$. Then for any $x \in \overline{x_{0} z}$, we define the sets $S_{x}$ and $\bar{S}_{x}$ in $X$ by

$$
S_{x}=\{r x \mid-\infty<r<1\}
$$

and

$$
\bar{S}_{x}=\{r x \mid-\infty<r \leq 1\}
$$

Let $x_{1}, x_{2} \in \overline{x y}$ be arbitrary. We say $x_{1}<x_{2}$ if $S_{x_{1}} \subset S_{x_{2}}$, or equivalently, $\overline{x_{0} x_{1}} \subset \overline{x_{0} x_{2}}$. In this case we also write $x_{2}>x_{1}$.

Let $M$ denote the $\sigma$-algebra of all subsets of $X$ such that

$(X, M)$ is a measurable space. Let $\mathrm{ca}(X, M)$ be the space of all vector measures (real signed measures) and define a norm $\|\cdot\|$ on $\operatorname{ca}(X, M)$ by

$$
\|\mathrm{p}\|=|\mathrm{p}|(\mathrm{X})
$$

where $|p|$ is a total variation measure of $p$ and is given by

$$
|p|(X)=\sup \sum_{i=1}^{\infty}\left|p\left(E_{i}\right)\right|, \quad E_{i} \subset X,
$$

Where the supremum is taken over all possible partitions $\left\{E_{i}: i \in N\right\}$ of $X$. It is known that $c a(X, M)$ is a Banach space with respect to the norm $\|$.$\| given by (4).$

Let $\mu$ be a $\sigma$-finite positive measure on $X$, and let $p \in c a(X, M)$. We say $p$ is absolutely continuous with respect to the measure $\mu$ if $\mu(E)=0$ implies $p(E)=0$ for some $E \in M$. In this case we also write $p<<\mu$.

Let $x_{0} \in X$ be fixed and let $M_{0}$ denote the $\sigma$-algebra on $S_{x_{0}}$. Let $z \in X$ be such that $z>x_{0}$ and let $M_{z}$ denote the $\sigma$ - algebra of all sets containing $M_{0}$ and the sets of the form $S_{x}, x \in \overline{x_{0} z}$.

Throughout this paper, unless otherwise mentioned, let $(E, \preceq,\|?\|)$ denote a partially ordered normed linear space. Two elements $x$ and $y$ in $E$ are said to be comparable if either the relation $x \preceq y$ or $y \preceq x$ holds. A non-empty subset $C$ of $E$ is called a chain or totally ordered if all the elements of $C$ are comparable. It is known that $E$ is regular if $\left\{x_{n}\right\}$ is a non decreasing (resp. non increasing) sequence in $E$ such that $x_{n} \rightarrow x^{*}$ as $n \rightarrow \infty$, then $x_{n} \preceq x^{*}$ (resp. $x_{n} \succeq x^{*}$ ) for all $n \in \mathrm{N}$. The conditions guaranteeing the regularity of $E$ may be found in Heikkiländand Lakshmikantham [8] and the references therein. We need the following definitions (see Dhage [14] and the references therein) in what follows.

Definition 3.1. A mapping $T: E \rightarrow E$ is called isotone or non-decreasing if it preserves the order relation $\preceq$, that is, if $x \preceq y$ implies $T x \preceq T y$ for all $x, y \in E$. Similarly, $T$ is called nonincreasing if $x \preceq y$ implies $T x \succeq T y$ for all $x, y \in E$. Finally, $T$ is called monotonic or simply monotone if it is either non decreasing non increasing on $E$.

Definition 3.2. A mapping $T: E \rightarrow E$ is called partially continuous at a point $a \in E$ if for $\varepsilon>0$ there exists a $\delta>0$ such that $\|T x-T a\|<\varepsilon$ whenever $x$ is comparable to $a$ and $\|x-a\|<\delta$. $T$ called partially continuous on $E$ if it is partially continuous at every point of it. It is clear that if $T$ is partially continuous on $E$, then it is continuous on every chain $C$ contained in $E$.

Definition 3.3. A non-empty subset $S$ of the partially ordered Banach space $E$ is called partially bounded if every chain $C$ in $S$ is bounded. An operator $T$ on a partially normed linear space $E$ into itself is called partially bounded if $T(E)$ is a partially bounded subset of $E$. $T$ is called uniformly partially bounded if all chains $C$ in $T(E)$ are bounded by a unique constant.

Definition 3.4. A non-empty subset $S$ of the partially ordered Banach space $E$ is called partially compact if every chain $C$ in $S$ is a relatively compact subset of $E$. A mapping $T: E \rightarrow E$ is called partially compact if $T(E)$ is a partially relatively compact subset of $E$. $T$ is called uniformly partially compact if $T$ is a uniformly partially bounded and partially compact operator on $E$. $T$ is called partially totally bounded if for any bounded subset $S$ of $E, T(S)$ is a partially relatively compact subset of $E$. If $T$ is partially continuous and partially totally bounded, then it is called partially completely continuous on $E$.

Definition 3.5. An upper semi-continuous and monotone non decreasing function $\psi: R_{+} \rightarrow R_{+}$is called a $D$-function provided $\psi(0)=0$. An operator $T: E \rightarrow E$ is called partially nonlinear $D$-contraction if there exists a $D$-function $\psi$ such that

$$
\|T x-T y\| \leq \psi(\|x-y\|)
$$

For all comparable elements $x, y \in E$, where $0<\psi(r)<r$ for $\mathrm{r}>0$. In particular, if $\psi(r)=k r, k>0, T$ is called a 
partial Lipschitz operator with a Lipschitz constant $k$ and more over, if $0<k<1, T$ is called a partial linear contraction on $E$ with a contraction constant $k$.

The Dhage iteration method or Dhage iteration principle embodied in the following applicable hybrid fixed point theorem of Dhage [12] in a partially ordered normed linear space is used as a key tool for our work contained in this paper. The details of the Dhage iteration method or principle is given in Dhage $[14,15]$, and the references therein.

Theorem 3.1. Let $(E, \preceq,\|?\|)$ be a regular partially ordered complete normed linear space such that every compact chain $\mathrm{C}$ of E. Let A,B: $E \rightarrow E$ be two nondecreasing operators such that

a) $\mathrm{A}$ is partially bounded and partially nonlinear Dcontraction,

b) $\mathrm{B}$ is partially continuous and partially compact, and

c) there exists an element $x_{0} \in E$ such that $x_{0} \preceq A x_{0}+B x_{0}$ or $x_{0} \succeq A x_{0}+B x_{0}$.

Then the operator equation $\mathrm{A} x+\mathrm{B} x=x$ has a solution $x^{*}$ in $\mathrm{E}$ and the sequence $\left\{\mathrm{x}_{\mathrm{n}}\right\}$ of successive iterations defined by

$x_{\mathrm{n}+1}=\mathrm{A} x_{\mathrm{n}}+\mathrm{B} x_{\mathrm{n}}, \mathrm{n}=0,1, \ldots$, converges monotonically to $x^{*}$.

\section{Main Result}

In this section, we prove an existence and approximation result for the AMDE (9) on a closed and bounded interval

$J=[a, b]$ under mixed partial Lipschitz and partial compactness type conditions on the nonlinearities involved in it. We place the AMDE (9) in the function space $C(J, R)$ of continuous real-valued functions defined on $J$. We define a norm $\|\cdot\|$ and the order relation $\leq$ in $C(J, R)$ by

$$
\|x\|=\sup _{t \in J}|x(t)|
$$

and

$$
x \leq y \Leftrightarrow x(t) \leq y(t) \text { for all } t \in J
$$

Clearly, $C(J, R)$ is a Banach space with respect to above supremum norm and also partially ordered w. r. t. the above partially order relation $\leq$. It is known that the partially ordered Banach space $C(J, R)$ is regular and lattice so that every pair of elements of $E$ has a lower and an upper bound in it.

Consider the first order ordinary nonlinear abstract measure differential equation,

$$
\left.\begin{array}{l}
\frac{d p}{d \mu}=f\left(x, p\left(S_{x}\right)\right), \text { a.e }[\mu], x \in \overline{x_{0} z} \\
p(E)=q(E), E \in M_{0}
\end{array}\right\}
$$

for all $x \in \overline{x_{0} z}$, where $\mu, f: s_{z} \times R \rightarrow R$ are continuous functions. Where $q$ is a given known vector measure, $\frac{d p}{d \mu}$ is a Radon-Nikodym derivative of $p$ with respect to $\mu, f: S_{z} \times R \rightarrow R$, and $f\left(x, p\left(S_{x}\right)\right)$ is $\mu$-integrable for each $p \in c \mathrm{a}\left(S_{z}, M_{z}\right)$.

By a solution of equation (9) we mean a differentiable function $p \in c a\left(S_{z}, M_{z}\right)$ that satisfies equation (9), where $C$ $(X, M)$ is the space of continuous real-valued functions defined on $\overline{x_{0} z}$. The equation (9) has already been discussed for different aspects of the solutions using the usual Picard iteration method. See Bainov and Hristova [11] and the references therein for the details. In this paper we discuss the AMDE (9) for existence and approximation of solutions via a new approach based upon the Dhage iteration method.

We need the following definition as follows.

Definition 4.1. A function $u \in c a\left(S_{z}, M_{z}\right)$ is said to be a lower solution of the AMDE (9) if it satisfies

$$
\left.\begin{array}{l}
\frac{d u}{d \mu} \leq f\left(x, p\left(S_{x}\right)\right), x \in \overline{x_{0} z} \\
p(E)=q(E), E \in M_{0}
\end{array}\right\}
$$

for all $x \in \overline{x_{0} z}$. Similarly, an upper solution $v \in c a\left(S_{z}, M_{z}\right)$ to the $\operatorname{AMDE}(9)$ is defined on $\overline{x_{0} z}$.

We consider the following hypotheses as follows:

$\left(\mathrm{H}_{1}\right)$ There exists a real number $\lambda>0$ such that the function $x \mapsto f\left(x, p\left(S_{x}\right)\right)+\lambda x$ is monotone non-decreasing for each $x \in \overline{x_{0} z}$.

$\left(\mathrm{H}_{2}\right)$ The AMDE(9) has a lower solution $u \in c a\left(S_{z}, M_{z}\right)$.

$\left(\mathrm{H}_{3}\right)$ There exists a constant $\mathrm{K}>0$ such that $\left|\tilde{f}\left(x, p\left(S_{x}\right)\right)\right| \leq K$, for all $x \in \overline{x_{0} z}$

Consider the following abstract measure differential equation

$$
\left.\begin{array}{rl}
\frac{d p}{d \mu}+\lambda p\left(S_{x}\right) & =\tilde{f}\left(x, p\left(S_{x}\right)\right), x \in \overline{x_{0} z} \\
p(E) & =q(E), E \in M_{0}
\end{array}\right\}
$$

where $\tilde{f}: S_{z} \times R \rightarrow R$ is defined by

$$
\tilde{f}\left(x, p\left(S_{x}\right)\right)=f\left(x, p\left(S_{x}\right)\right)+\lambda p\left(S_{x}\right), \lambda>0 .
$$

Remark 4.1. A vector measure $u \in c a\left(S_{z}, M_{z}\right)$ is a solution of the equation (13) if and only if it is a solution of the equation (9) defined on $\overline{x_{0} z}$.

Lemma 4.1. Assume that hypothesis $\left(\mathrm{H}_{3}\right)$ holds. Then a function $u \in c a\left(S_{z}, M_{z}\right)$ is a solution of the AMDE (9) if and only if it is a solution of the nonlinear integral equation,

$$
p(x)=x_{0} e^{-\lambda x}+e^{-\lambda x} \int_{0}^{x} e^{\lambda x} \tilde{f}\left(x, p\left(S_{x}\right)\right) d x
$$

for all $x \in \overline{x_{0} z}$.

Theorem 4.1. Assume that hypotheses $\left(\mathrm{H}_{1}\right)$ through $\left(\mathrm{H}_{3}\right)$ 
hold. Then the AMDE (9) has a solution $x^{*}$ defined on $\overline{x_{0} z}$ and the sequence $\left\{p_{n}\right\}_{n=1}^{\infty}$ of successive approximations defined by

$$
p_{n+1}(x)=x_{0} e^{-\lambda x}+e^{-\lambda x} \int_{0}^{x} e^{\lambda x} \tilde{f}\left(x, p_{n}(x)\right) d x, x \in \overline{x_{0} z}
$$

where $x_{0}=u, x \in \overline{x_{0} z}$, converges monotonically to $x^{*}$.

Proof. Set $E=c a\left(S_{z}, M_{z}\right)$. Then, in view of Lemma 3.1, every compact chain $C$ in $E$ possesses the compatibility property with respect to the norm $\|$.$\| and the order relation \leq$ in $E$.

Define the operator $T$ on $E$ by

$$
T p(x)=x_{0} e^{-\lambda x}+e^{-\lambda x} \int_{0}^{x} e^{\lambda x} \tilde{f}\left(x, p\left(S_{x}\right)\right) d x, x \in \overline{x_{0} z}
$$

From the continuity of the integrals, it follows that $T$ defines the functions $T: E \rightarrow E$. Now by Lemma 4.1, the AMDE (9) is equivalent to the operator equation

$$
T p(x)=p(x), x \in \overline{x_{0} z}
$$

We shall show that the operator $T$ satisfies all the conditions of Theorem 3.1. This is achieved in the series of following steps.

Step I: $T$ is a non decreasing operator on $E$.

Let $x, y \in E$ be such that $x \geq y$. Then by hypothesis $\left(\mathrm{H}_{1}\right)$, we obtain

$$
\begin{aligned}
T p(x) & =x_{0} e^{-\lambda x}+e^{-\lambda x} \int_{x_{0}}^{x} e^{\lambda x} \tilde{f}\left(x, p\left(S_{x}\right)\right) d x \\
& \geq x_{0} e^{-\lambda x}+e^{-\lambda x} \int_{x_{0}}^{x} e^{\lambda x} \tilde{f}\left(x, p\left(S_{y}\right)\right) d x \\
& =T p(y),
\end{aligned}
$$

for all $x \in \overline{x_{0} z}$. This shows that $T$ is a non decreasing operator on $E$ into $E$.

Step II: $T$ is a partially continuous operator on $E$.

Let $\left\{p_{n}\right\}$ be a sequence in a chain $C$ in $E$ such that $p_{n} \rightarrow p$ for all $n \in N$.

Then, by dominated convergence theorem, we have

$$
\begin{aligned}
\lim _{n \rightarrow \infty} T p_{n}(x) & =\lim _{n \rightarrow \infty}\left[x_{0} e^{-\lambda x}+e^{-\lambda x} \int_{0}^{x} e^{\lambda x} \tilde{f}\left(x, p_{n}\left(S_{x}\right)\right) d x\right] \\
& =x_{0} e^{-\lambda x}+e^{-\lambda x} \int_{0}^{x} e^{\lambda x}\left[\lim _{n \rightarrow \infty} \tilde{f}\left(x, p_{n}\left(S_{x}\right)\right)\right] d x \\
& =x_{0} e^{-\lambda x}+e^{-\lambda x} \int_{0}^{x} e^{\lambda x}\left[\tilde{f}\left(x, p_{n}\left(S_{x}\right)\right)\right] d x \\
& =T p(x),
\end{aligned}
$$

for all $x \in \overline{x_{0} z}$. This shows that $T p_{n}$ converges to $T p$ pointwise on $\overline{x_{0} z}$.

Next, we will show that $\left\{T p_{n}\right\}$ is an equicontinuous sequence of functions in $E$. Let $x_{1}, x_{2} \in \overline{x_{0} z}$ be arbitrary with $x_{1}<x_{2}$. Then

$$
\begin{aligned}
\left|T p_{n}\left(x_{2}\right)-T p_{n}\left(x_{1}\right)\right| & =\left|e^{-\lambda x_{2}} \int_{0}^{x_{2}} e^{\lambda x} \tilde{f}\left(x, p_{n}\left(S_{x}\right)\right) d x-e^{-\lambda x_{1}} \int_{0}^{x_{1}} e^{\lambda x} \tilde{f}\left(x, p_{n}\left(S_{x}\right)\right) d x\right| \\
& \leq\left|\left(e^{-\lambda x_{2}}-e^{-\lambda x_{1}}\right) \int_{0}^{x_{1}} e^{\lambda x} \tilde{f}\left(x, p_{n}\left(S_{x}\right)\right) d x\right|+\left|e^{-\lambda x_{2}} \int_{x_{1}}^{x_{2}} e^{\lambda x} \tilde{f}\left(x, p_{n}\left(S_{x}\right)\right) d x\right| \\
& \leq\left|\left(e^{-\lambda x_{2}}-e^{-\lambda x_{1}}\right) \int_{0}^{T} e^{\lambda x} K d x\right|+\left|\int_{x_{1}}^{x_{2}} e^{\lambda x} K d x\right| \\
& \rightarrow 0 \text { as } \quad x_{1} \rightarrow x_{2}
\end{aligned}
$$

uniformly for all $n \in N$. This shows that the convergence $T p_{n} \rightarrow T p$ is uniformly and hence $T$ is a partially continuous operator on $E$ into itself.

Step III: $T$ is a partially compact operator on E.

Let $C$ be an arbitrary chain in $E$. We show that $T(C)$ is a uniformly bounded and equicontinuous set in $E$. First we show that $T(C)$ is uniformly bounded. Let $x \in C$ be arbitrary. Then,

$$
\begin{aligned}
|T p(x)| & \leq\left|x_{0} e^{-\lambda x}\right|+\left|e^{-\lambda x} \int_{0}^{x} e^{\lambda x} \tilde{f}\left(x, p\left(S_{x}\right)\right) d x\right| \\
& \leq\left|x_{0}\right|+\int_{0}^{x} e^{\lambda x}\left|\tilde{f}\left(x, p\left(S_{x}\right)\right)\right| d x \mid \\
& \leq\left|x_{0}\right|+\int_{0}^{T} e^{\lambda T} K d x \\
& \leq\left|x_{0}\right|+e^{\lambda T} K T=r,
\end{aligned}
$$

for all $x \in \overline{x_{0} z}$. Taking supremum over $x$, we obtain $\|T x\| \leq r$ for all $x \in C$. Hence $T$ is a uniformly bounded subset of $E$. Next, we will show that $T(C)$ is an equicontinuous set in $E$. Let $x_{1}, x_{2} \in \overline{x_{0} z}$ be arbitrary with

$$
x_{1}<x_{2} \text {. Then }
$$

$$
\begin{aligned}
\left|T p\left(x_{2}\right)-T p\left(x_{1}\right)\right| & =\left|e^{-\lambda x_{2}} \int_{0}^{x_{2}} e^{\lambda x} \tilde{f}\left(x, p\left(S_{x}\right)\right) d x-e^{-\lambda x_{1}} \int_{0}^{x_{1}} e^{\lambda x} \tilde{f}\left(x, p\left(S_{x}\right)\right) d x\right| \\
& \leq\left|\left(e^{-\lambda x_{2}}-e^{-\lambda x_{1}}\right) \int_{0}^{x_{1}} e^{\lambda x} \tilde{f}\left(x, p\left(S_{x}\right)\right) d x\right|+\left|e^{-\lambda x_{2}} \int_{x_{1}}^{x_{2}} e^{\lambda x} \tilde{f}\left(x, p\left(S_{x}\right)\right) d x\right| \\
& \leq\left|\left(e^{-\lambda x_{2}}-e^{-\lambda x_{1}}\right) \int_{0}^{T} e^{\lambda x} K d x\right|+\left|\int_{x_{1}}^{x_{2}} e^{\lambda x} K d x\right| \\
& \rightarrow 0 \text { as } x_{1} \rightarrow x_{2}
\end{aligned}
$$

uniformly for all $x \in C$. Hence $T(C)$ is a compact subset of $E$ and consequently $T$ is a partially compact operator on $E$ into itself.

Step IV: $u$ satisfies the operator inequality $u \leq T u$.

By hypothesis $\left(\mathrm{H}_{2}\right)$, the AMDE (9) has a lower solution $u$. Then we have 


$$
\left.\begin{array}{l}
\left.\frac{d u}{d \mu} \leq f\left(x, u\left(S_{x}\right)\right)\right), \\
u(0) \leq x_{0}
\end{array}\right\}
$$

for all $x \in \overline{x_{0} z}$ Adding $\lambda u\left(S_{x}\right)$ on both sides of the first inequality in (16), we obtain

$$
\frac{d u}{d \mu}+\lambda u\left(S_{x}\right) \leq f\left(x, u\left(S_{x}\right)\right)+\lambda u\left(S_{x}\right), x \in \overline{x_{0} z} .
$$

Again, multiplying the above inequality (17) by $e^{\lambda x}$,

$$
\left(e^{\lambda x} u\left(S_{x}\right)\right)^{\prime} \leq e^{\lambda x} \tilde{f}\left(x, u\left(S_{x}\right)\right)
$$

A direct integration of (18) from 0 to $x$ yields

$$
u\left(S_{x}\right) \leq x_{0} e^{-\lambda x}+e^{-\lambda x} \int_{0}^{x} e^{\lambda x} \tilde{f}\left(x, u\left(S_{x}\right)\right) d x
$$

for all $x \in \overline{x_{0} z}$. From definition of the operator $T$ it follows that

$$
u\left(S_{x}\right) \leq T u\left(S_{x}\right)
$$

for all $x \in \overline{x_{0} z}$. Hence $u \leq T u$.

Thus $T$ satisfies all the conditions of Theorem 3.1 and we apply it to conclude that the operator equation $T x=x$ has a solution. Consequently the integral equation and the AMDE (9) has a solution $x^{*}$ defined on $\overline{x_{0} z}$. Furthermore, the sequence $\left\{p_{n}\right\}$ of successive approximations defined by (1) converges monotonically to $p^{*}$. Hence the proof.

Remark 4.2. The conclusion of Theorem 4.1 also remains true for an upper solution $v \in c a\left(S_{z}, M_{z}\right)$.

Example 4.1. Consider the following abstract differential equation,

$$
\left.\begin{array}{c}
\frac{d p}{d \mu}=\tan ^{-1} p\left(S_{x}\right)-p\left(s_{x}\right), \\
x(0)=1
\end{array}\right\}
$$

for all $x \in \overline{x_{0} z}$

Here, $f\left(x, p\left(S_{x}\right)\right)=\tan ^{-1} x-x$. Clearly, the functions $\mathrm{f}$ is continuous on $S_{z} \times R$. The function $\mathrm{f}$ satisfies the hypothesis $\left(\mathrm{H}_{1}\right) \quad$ with $\lambda=1$. Moreover, the function $\tilde{f}\left(x, p\left(S_{x}\right)\right)=\tan ^{-1} x$ is bounded on $S_{z} \times R$ with bound $K=\frac{\pi}{2}$ and so the hypothesis $\left(\mathrm{H}_{3}\right)$ is satisfied.

Since for all $x \in R$, any function $u \in c a\left(S_{z}, M_{z}\right)$ satisfying the linear differential equation

$$
\left.\begin{array}{c}
\frac{d p}{d \mu}+p\left(S_{x}\right)=-2, \\
x(0)=1,
\end{array}\right\}
$$

is a lower solution of the AMDE (14) on $\overline{x_{0} z}$. Because, in this case, we obtain

$$
\left.\begin{array}{c}
\frac{d p}{d u}+u\left(S_{x}\right) \leq \tan ^{-1} u\left(S_{x}\right) \\
x(0)=1
\end{array}\right\}
$$

for all $x \in \overline{x_{0} z}$. Therefore, solving (21) for unknown function $u$, we get

$$
u(x)=3 e^{-x}-2, x \in \overline{x_{0} z} .
$$

Similarly, any function $v \in c a\left(S_{z}, M_{z}\right)$ satisfying the linear differential equation

$$
\left.\begin{array}{c}
\frac{d p}{d \mu}+p\left(S_{x}\right)=-2, \\
x(0)=1,
\end{array}\right\}
$$

is an upper solution of the AMDE (4.14) on $\overline{x_{0} z}$. Solving the differential equation (4.18) for the unknown function $v$ then we get

$$
v(x)=2-e^{-x}, x \in \overline{x_{0} z} .
$$

Hence, we apply Theorem 4.1 and conclude that the AMDE (20) has a solution $x^{*}$ defined on $\overline{x_{0} z}$ and the sequence $\left\{p_{n}\right\}_{n=1}^{\infty}$ of successive approximations defined by

$$
p_{n+1}(x)=e^{-x}+e^{-x} \int_{0}^{x} e^{x} \tan ^{-1} p_{n}\left(S_{x}\right) d x
$$

for all $x \in \overline{x_{0} z}$, where $p(x)=3 e^{-x}-2, x \in \overline{x_{0} z}$, converges monotonically to $\mathrm{x} *$.

Remark 4.3. The existence of solutions $x^{*}$ of the AMDE (20) may be obtained under the assumption of existence of the upper solution $v$ defined on $\overline{x_{0} z}$. Here also we conclude that the AMDE (20) has a solution $x^{*}$ defined on $\overline{x_{0} z}$ and the sequence $\left\{p_{n}\right\}$ of successive approximations defined by (26) with $x_{1}=2-e^{-x}$ converges monotonically to $x^{*}$.

\section{Conclusion}

It is clear that the Dhage iteration method is a powerful tool for proving the existence and approximating results for nonlinear measure differential equations but with some limitations in Picard's method. It is very simple in nature for the solution under weaker partial Lipschitz and compactness conditions. 


\section{References}

[1] M. A. Krasnoselskii, Topological Methods in the Theory of Nonlinear Integral Equations, Pergamon Press, New York, 1964.

[2] R. R. Sharma, An Abstract Measure Differential Equations, proc. Amer. Math. Soc. 32 (1972) 503-510.

[3] S. R. Joshi, A system of abstract measure delay differential equations J. Math. Phys. Sci. 13 (1979) 497-506.

[4] G. R. Shendge, S. R. Joshi, Abstract measure differential inequalities and applications, Acta Math. Hun 41 (1983) 53-54.

[5] B. C. Dhage, On abstract measure integro-differential equations. J. Math. Phys. Sci. 20 (1986) 367-380.

[6] B. C. Dhage On system of abstract measure integrodifferential inequalities and applications, Bull Inst. Math. Acad. Sin. 18 (1989), 53-54.

[7] S. Heikkiläand, V. Lakshmikantham, Monotone Iterative Techniques for Discontinuous Nonlinear Differential Equations, Marcel Dekker Inc., New York 1994.

[8] G. A. Granas. J. Dugundji, Fixed Point Theory, Springer Verlag. 2003.

[9] B. C. Dhage, S. S. Bellale, Abstract measure integrodifferential equations, Global J. Math. Anal. 1 (1-2) (2007) 91-108

[10] B. C. Dhage, and S. S. Bellale, Existence theorem for perturbed Abstract measure differential equations. Nonlinear Analysis 71 (2009) 319-328.

[11] D. D. Bainov, S. Hristova, Differential Equations with Maxima, Chapman \& Hall/CRC Pure andApplied Mathematics, 2011.
[12] B. C. Dhage, Hybrid fixed point theory in partially ordered normed linear spaces and applications to fractional integral equations, Differ. Equ Appl., 5 (2013), 155-184.

[13] S. S. Bellale, Hybrid Fixed point theorem for abstract measure differential equation, World Academy Of Science, Engineering and Technology, 73 (2013) 782-785.

[14] B. C. Dhage, Approximating Solutions of nonlinear first order ordinary differential equations, Global Journal of mathematical sciences 3 (2014), 1-9.

[15] B. C. Dhage, A new monotone iteration principle in the theory of nonlinear first order integro differential equations, Nonlinear Studies, 22 (3) (2015), 397-417.

[16] S. B. Dhage, A. D. Kadam, Dhage Iteration method for initial value problems of nonlinear second order hybrid functional differential equations. Electronic Journal of Mathematical Analysis and Applications Vol (1), Jan. 2018 pp. 79-93.

[17] D. M. Suryawanshi, S. S. Bellale, Dhage Iteration Method for Non-Linear first order abstract measure integro differential equations with linear perturbation. International Journal of Mathematics Trends and Technology (IJMTT)-Volume 64 issue 2 Dec 2018, 115-129.

[18] D. M. Suryawanshi, S. S. Bellale, Dhage Iteration Method for Non-Linear first order abstract measure differential equations with linear perturbation. International Journal of Mathematics Trends and Technology (IJMTT)-Volume 65 issue 2 Feb 2019, 139-149.

[19] D. M. Suryawanshi, S. S. Bellale, Iteration method for approximating solutions of perturbed abstract measure differential equations. Journal of Emerging Technologies and Innovative. April 2019, 746-752. 